\title{
Direct numerical simulation of compressible free shear flows
}

\author{
By S. K. LELE
}

\begin{abstract}
Direct numerical simulations of compressible free shear layers in open domains are conducted. Compact finite-difference schemes of spectral-like accuracy are used for the simulations. Both temporally-growing and spatially-growing mixing layers are studied. The effect of intrinsic compressibility on the evolution of vortices is studied. The use of convective Mach number is validated. Details of vortex roll up and pairing are studied. A simple explanation of the stabilizing effect of compressibility is offered. Acoustic radiation from vortex roll up, pairing and shape oscillations is studied and quantified.
\end{abstract}

\section{Introduction}

Direct numerical simulations have been used to study several incompressible turbulent flows. These studies have ranged from the simulations of homogeneous turbulence (Rogallo, 1981, Lee and Reynolds, 1985, and Rogers, Moin and Reynolds, 1986), to the turbulence in channel flows, (Moser and Moin, 1987, and Kim, Moin and Moser, 1987), boundary layers, (Spalart, 1988), and free shear flows, (Corcos and Sherman, 1984, Corcos and Lin, 1984, Riley and Metcalfe, 1980, Metcalfe et. al., 1987, Lowery and Reynolds, 1986, and Sandham and Reynolds, 1987). The results obtained from the simulations have been extensively compared with the experimental measurements (Kim, Moin and Moser, 1987, Spalart, 1988) and are being used as databases for developing and testing turbulence models (Mansour, Kim and Moin, 1988, and Center for Turbulence Research, 1987), studying the structure and dynamics of organized motions in turbulent flows (Center for Turbulence Research, 1987 and 1988), and testing the accuracy of experimental techniques by Moin and Spalart (1987). On the other hand, the study of compressible turbulent flows is relatively less mature. Most simulations of compressible flows have been limited to the simulation of the large scales of the flow (Winkler et. al., 1987, Smarr et. al., 1984, Woodward, 1984, and Boris, 1988), with some notable exceptions (Feiereisen, Reynolds and Ferziger, 1981, and Passot and Pouquet, 1987). Comparisons with experimental measurements are less detailed in part due to the 'large scale' and two-dimensional nature of most simulations and lack of experimental data on turbulent fluctuations in compressible flows. The renewed interest in the high speed flows, as well as the need to develop turbulence models which incorporate the compressibility effects provide the motivation for the present work. This 
paper provides a brief account of results obtained from direct simulations of compressible free shear layers. The simulations resolve all the relevant scales of motion and in particular do not employ any form of filtering or artificial dissipation. Simulations are conducted for both two-dimensional and three-dimensional flows. Most results presented are based on the two-dimensional simulations.

\section{Numerical Method}

The numerical method used in the study is a compact finite difference scheme of spectral-like accuracy. The details of the numerical scheme have been described in detail elsewhere (Lele, 1988a). The full unsteady equations for mass, momentum and energy conservation are solved for an ideal gas. The Newtonian viscosity law (zero bulk viscosity), and Fourier law of heat conduction are used. Variation of viscosity and thermal conductivity with temperature is permitted. Conservation equation for of a passive scalar (mass fraction) is also solved. Time advancement is carried out by a compact storage third order Runge-Kutta scheme developed by Wray (1987).

The main features of the finite-difference scheme are summarized. The finite difference approximation $f_{i}^{\prime}$ to the first derivative $\frac{d f}{d x}\left(x_{i}\right)$ at the node $i$ is evaluated by solving a tridiagonal system of the form:

$$
\alpha f_{i-1}^{\prime}+f_{i}^{\prime}+\alpha f_{i+1}^{\prime}=b \frac{f_{i+2}-f_{i-2}}{4 h}+a \frac{f_{i+1}-f_{i-1}}{2 h} .
$$

The relations between the coefficients $a, b$ and $\alpha$ are derived by matching the Taylor series coefficients of various order. With

$$
a=\frac{2}{3}(\alpha+2), b=\frac{1}{3}(4 \alpha-1)
$$

a family of fourth order schemes is obtained. It may be noted that as $\alpha \rightarrow 0$ this family merges into the well known fourth order central difference scheme. Similarly for $\alpha=\frac{1}{4}$ the classical Pade' scheme is recovered. Furthermore for $\alpha=\frac{1}{3}$ the leading order truncation error coefficient vanishes and the scheme is formally sixth order accurate. Most of the simulations presented here use this sixth order scheme. The spectral-like accuracy of the scheme follows from the nature of the dispersive errors associated with (1.1). It may be shown that compared to the traditional finite difference schemes the scheme (1.1) reduces the dispersive errors over a wider band of the length scales represented on the grid. This characterization of the dispersive errors is presented in figure-1. The straight line on the figure represents a spectrally accurate scheme (i.e. exact for all the wavenumbers represented on the mesh). The improved representation of the shorter spatial scales by the present scheme is evident from the figure.

If the dependent variables are periodic then the system of relations (1.1) written for each node can be solved together as a linear system of equations. The 
general non-periodic case requires additional relations appropriate for the near boundary nodes. These are described in Lele (1988a). The relation (1.1) along with a mathematically defined mapping between a non-uniform physical mesh and a uniform computational mesh provides derivatives on a non-uniform mesh.

The second derivatives are evaluated by solving a system similar to (1.1), viz. $\alpha f_{i-1}^{\prime \prime}+f_{i}^{\prime \prime}+\alpha f_{i+1}^{\prime \prime}=$

$$
b \frac{f_{i+2}-2 f_{i}+f_{i-2}}{4 h^{2}}+a \frac{f_{i+1}-2 f_{i}+f_{i-1}}{h^{2}}
$$

where $f_{i}^{\prime \prime}$ represents the finite difference approximation to the second derivative at node $i$. With

$$
a=\frac{4}{3}(1-\alpha), b=\frac{1}{3}(-1+10 \alpha),
$$

a one parameter family of fourth order schemes is obtained. Again as $\alpha \rightarrow 0$ this family coincides with the well known fourth order central difference scheme. For $\alpha=\frac{1}{10}$ the classical Pade' scheme is recovered. For $\alpha=\frac{2}{11}$ a sixth order tridiagonal scheme is obtained. This scheme with

$$
\alpha=\frac{2}{11}, a=\frac{12}{11}, b=\frac{3}{11}
$$

is used for the simulations presented here. It may also be noted that the schemes (1.3) provide an accurate evaluation of the second derivative over a wide range of length scales. The error associated with the second derivative evaluation for a variety of schemes is shown on figure-2. The spectrally accurate evaluation corresponds to the parabola on the figure. The improvement of the present scheme in representing the shorter scales is again evident from this figure.

Simulations are conducted for both spatially-evolving and temporally-evolving mixing layers. In both cases non-reflecting boundary conditions (Thompson, 1987) are employed at the top and bottom boundaries of the computational domain. In the spatially-evolving case non-reflecting boundary conditions are also used at the inflow and outflow boundaries. The computational grid is uniformly spaced in $x$ (mean flow direction) and $z$ (spanwise direction) and non-uniform in $y$ (transverse to the mean flow). Typically a hyperbolic tangent mapping is used for the non-uniform mesh (in $y$ ) with the maximum grid spacing about 2-4 times the minimum grid spacing.

\section{Mixing Layer Flows}

Direct numerical simulations of mixing layer forming between two streams flowing with speeds $U_{1}$ (faster stream) and $U_{2}$ were conducted. The two streams were matched in static pressure but, in general, had different fluid densities. Such two stream mixing layers were studied by Brown and Roshko (1974) and subsequently by other investigators. They noted that while the density ratio of the 
two streams had a noticeable effect on the spreading rate of the mixing layer it was not enough to explain the slow spreading rate of supersonic mixing layers. Papamoschou and Roshko (1986) (referred to as PR from hereon) showed that this compressibility effect on the spreading rate could be parameterized in terms of a single Mach number, the Convective Mach Number $M_{c}$, viz. the Mach number defined in a frame of reference moving with the dominant eddies of the flow. They also derived an expression for $M_{c}$ in terms of the flow conditions of the two streams. Bogdanoff (1983) also suggested the same 'intrinsic Mach number' by a different argument. Recent experiments (Papamoschou, 1988, and Samimy and Elliott, 1988) stability analyses (Sandham and Reynolds, 1989, Ragab and Wu, 1988, Zhuang et. al., 1988) and numerical simulations (Soetrisno et. al., 1988 , Lele 1988b) have provided further support to the this notion. Numerical simulations are used to test the notion of the convective Mach number in a fundamental way. Simulations were conducted for three types of mixing layer flows: A) Mixing of streams of equal entropy, B) Mixing of streams of equal stagnation enthalpy, and C) Mixing of streams of equal Mach number. These different classes of mixing layer flows allowed us to vary the Mach number of the individual streams while keeping the convective Mach number fixed. It is thus possible to look for the influence of factors other than the convective Mach number. The spatially evolving simulations presented here were forced at the inflow by adding a v-velocity disturbance velocity of small amplitude (typically with a peak of $1 \%$ ) to the inflow profiles. This disturbance was confined to the shear layer by means of a Gaussian shape function. It is possible to refine the forcing by using the linear stability eigenfunctions of the inflow profiles. Such a approach was used by Sandham and Reynolds (1987) in simulating incompressible mixing layers. The inflow disturbance contained the most unstable frequency and its first two subharmonics.

In the temporally evolving simulations the initial condition provided the source of disturbance. Small amplitude incompressible disturbances were added to the tangent hyperbolic mean flow profile. Two groups of simulations were conducted. 1) Organized initial conditions - the disturbance contained a small number (typically two) of fourier modes with prescribed phase relations, and 2) Random initial conditions - the disturbance had a white noise wavenumber spectrum with very small (typically $10^{-4}$ or smaller) amplitude. Both $2-\mathrm{d}$ and 3-d simulations were conducted with the random initial conditions.

The simulations had specific heat ratio, $\gamma=1.4$, Prandtl number, $P r=3 / 4$, and Reynolds number $R=\frac{\rho_{1}\left(U_{1}-U_{3}\right) \delta_{0}}{\mu_{1}}$ in the range 100-500, where subscripts 1 and 2 refer to the high speed and low speed streams, respectively, and $\delta_{\omega_{0}}$ is the initial vorticity thickness.

\section{Intrinsic compressibility}

The definition of a convective Mach number requires one to specify the speed of propagation of the dominant eddies. This speed of propagation was determined 
in the numerical simulations from plots of the location of local pressure extremas against time. The pressure maxima correspond to the stagnation points between the vortices, and the pressure minima correspond to the vortex centers. The propagation speed is taken as the slope of these trajectories. An example of such a plot is shown in figure 3 for a class-A flow. For this particular case the Mach numbers of the two streams are 2.0 and 1.2 and the inflow disturbance contains the fundamental and its first two subharmonics. The trajectories of the stagnation points are shown in figure 3a. The location of the vortex centers are displayed in figure $3 \mathrm{~b}$. Two generations of pairing events can be seen in these figures. It may be noted that even with the pairing events the stagnation points move with a relatively uniform speed. During the pairing events the downstream vortex slows down while the upstream vortex speeds up. As the vortices pair the stagnation point in their middle is lost. It is also seen that the lower subharmonics tend to modulate the location of upstream pairing events. Varying the frequency of the fundamental disturbance did not alter the propagation speed of the vortices.

PR presented a formula for the vortex propagation speed based on the physical argument that the static pressure at the stagnation point is related to the stagnation pressures in the two streams by the isentropic relation. This convection speed formula was tested against the numerical simulation results at three different velocity ratios and several convective Mach numbers. The agreement between the observed speed and the formula is excellent in all the simulations (discrepancy of 1-2\%). It may be noted that when the specific heat ratio of the two gases is the same, the PR formula reduces to the formula proposed by Dimotakis (1984) for the propagation speed of vortices in incompressible shear layers. Thus fluid compressibility appears to have very little influence on the propagation of two-dimensional vortices.

The spatial growth rate of the mixing layer depends both on the growth measured in the convected frame of reference (temporal growth) and the convection speed of the vortices. Having verified that the convection speed is independent of the compressibility, compressibility effects in the spatial growth of the layer are studied. Figure 4 shows an example of the downstream evolution of three measures of mixing layer thickness. These are: 1) Thickness based on the Reynolds-averaged mean flow profile, 2) Thickness based on the Favre-averaged mean flow profile, and 3) Thickness based on the mean potential vorticity profile. For the example displayed, the layer was forced at the most unstable frequency with a $5 \%$ forcing amplitude. The three cases (one from each class) have $M_{c}$ of about 0.4 and the same velocity ratio $\frac{U_{2}}{U_{1}}$ of 0.6. The thickness is normalized by the initial thickness and the downstream distance is normalized by $10 \delta_{\omega_{0}}$. It may be seen when $M_{c}$ is the same the spatial growth rate for the three cases is similar but not identical. The differences that remain arise from the dependence of the nondimensional convection speed $\frac{U_{c}}{U_{1}}$ on the density ratio $\frac{\rho_{2}}{\rho_{1}}$. Case $\mathrm{C}$ has the slowest propagation speed giving the fastest spatial growth, while case $B$ has 
the fastest propagation speed and the slowest growth. A visual picture of the spatially evolving mixing layers is presented in figure-5. Contours of vorticity are plotted for three simulations with $M_{c}$ about 0.4 . The simulations were forced with the fundamental and two subharmonics. Vortex pairing events noted in figure-3 can be visually seen in these contours. It may also be noted that the vortices are most evolved in case $C$ (slowest propagation speed).

Results obtained from the vortex roll up in temporally growing mixing layers are presented to further verify this density ratio effect on $\frac{U_{c}}{V_{1}}$. The temporal simulations are conducted in a frame of reference moving with $U_{c}=\frac{a_{1} U_{2}+a_{2} U_{1}}{a_{1}+a_{2}}$, $a_{1}$ and $a_{2}$ being the sound speeds in the two streams. In this way the density ratio effect is removed. The initial amplitude in all the temporal simulations is 1 \%. In figure 6 two examples are shown with $M_{c}$ of about 0.4. The first case has streams with equal static temperature, i.e. $T_{2}=T_{1}$ (class A) and the second case has streams with equal total temperature, i.e. $h_{01}=h_{02}$ (class B). Thickness measures (1) and (2) defined above are displayed against time normalized by $\frac{10 \delta_{\omega_{0}}}{U_{1}-U_{c}}$. It may be noted that the time histories are almost identical.

Experiments have documented the reduction in the growth rate of compressible shear layers as $M_{c}$ is increased (PR). This reduction is most easily understood in terms of the behavior of the temporally-evolving layer. In figure 7 we show the time history of thickness measure (2) for several different $M_{c}$. The stabilizing influence of $M_{c}$ is clearly seen. It has been suggested that the slow growth arises primarily due to the reduced linear instability growth rate (PR and Sandham and Reynolds, 1989).

In the next section we present a physical argument for this stabilizing effect.

\section{Evolution of the vorticity field}

The linear instability process leads to a reorganization of the vorticity field. The inviscid vorticity equation for the two dimensional flow may be written as:

$$
\frac{\partial \omega}{\partial t}+U_{c} \frac{\partial \omega}{\partial x}=-\left(u-U_{c}\right) \frac{\partial \omega}{\partial x}-v \frac{\partial \omega}{\partial y}-\omega \nabla \cdot \vec{u}+\frac{\nabla \rho \times \nabla p}{\rho^{2}}
$$

showing that the rate of change of vorticity observed by an observer moving at speed $U_{c}$ is due to three effects. 1) Advection relative to the observer (first two terms on the r.h.s.), 2) Change due to compression of fluid elements (third term), and 3) Baroclinic change (fourth term). The individual terms for a class A mixing layer at $M_{c}$ of 0.4 are shown in figure 8. It may be noted that the most important term causing the vorticity redistribution is the advection term. Advection may be seen as moving vorticity away from the stagnation region and bringing it to the vortex centers. This is precisely the incompressible instability mechanism causing the shear layer instability. The compression term, while not dominant at $M_{c}=0.4$, provides clues on how the stabilizing effect associated 
with compressibility arises. A simple explanation of the stabilizing effect of compressibility is presented below. In figure 10 a schematic picture of the flow field around the vortices is presented in a frame of reference moving with speed $U_{c}$, the speed of the vortices. As shown earlier the 'stagnation points' between the vortices also move at the same speed and thus are stationary in this frame. Fluid moving away from the stagnation points expands as it accelerates towards the vortex center. Past this point the flow compresses and decelerates towards the other stagnation point. This expansion and compression process may be seen in the characteristic quadrupole pattern of the dilatation field. The expansion and compression cycle has the effect of increasing the vorticity near the stagnation points and reducing it near the vortex center. This is exactly opposite of the redistribution arising from the advection term (the driving cause of instability). The numerical simulations show that as $M_{c}$ is increased the vorticity compression effect becomes comparable in magnitude to the advection term.

In class $\mathbf{A}$ flows, the baroclinic change is an order of magnitude smaller than the other terms. In class $\mathrm{B}$ and $\mathrm{C}$ flows, the density of the two streams are unequal and this generates strong baroclinic torques. This is essentially an incompressible effect and arises whenever a density gradient exists across the shear layer. As the roll up proceeds this density interface remains sharp at the 'braids' and the pressure maximum (at the stagnation point in the convected frame) produces regions with dynamically significant baroclinic torque. For class B flows, the density of the low speed stream is always lower, thus the baroclinic torques tend to enhance the vorticity in the lower part of the braid and cause suppression of vorticity in the upper part of the braid. For class $C$ flows, the density of the high speed stream is lower and a reversal of the situation just described takes place. The vortices evolving in class $B$ and class $C$ flows have a layered vorticity distribution. The baroclinic effect strongly modifies the vorticity distribution in the vortices. This mechanism of generation of baroclinic torques is shown in figure 9 for a class $C$ flow. The net circulation of the vortices, however, remains relatively uninfluenced by this redistribution.

\section{Flows with eddy shocklets}

For $M_{c}$ around 0.7 and larger, the flow fields develop eddy shocklets. These shocks remain attached to the vortices and travel with them. For $M_{c}=0.7$ the shocks arise during the vortex pairing events, but the flow field is otherwise shock free. For $M_{c}=0.8$ the shocklets are produced during the roll up. An example of flow fields with eddy shocklets is displayed in figure 11. Just as observed at lower $M_{c}$ the flow accelerates and decelerates around the vortices. Now, however, there are local regions where the relative Mach number of unity is exceeded, and near the vortex this supersonic flow slows down by first going through a shock, becoming subsonic, and then decelerating further by compressing towards the stagnation points. The regions of expansion are now more spread out and the regions of compression more compact (essentially 
within the eddy shocklets). The increase of the vorticity due to the compression in the shock, as well as the increase in entropy is clearly observed. This flow field is remarkably similar to typical transonic flow past an airfoil. The induced velocity pattern associated with the fluid expansion and compression opposes the entrainment velocity induced by the clumped vorticity field. This may cause a further reduction in the entrainment of fluid into the layer.

\section{Acoustic radiation from vortex evolution}

Numerical simulations have been used to study the acoustic radiation arising from the unsteady processes of vortex roll up, pairing, shredding, and shape oscillations. The acoustic efficiency for these processes has been studied at several Mach numbers. At low Mach number less than $0.1 \%$ of the energy extracted from the mean flow is radiated. This fraction increases to $1-2 \%$ at $M_{c}$ of 0.6. A full account of these studies will be presented elsewhere (Lele and Ho, 1989). Here we present one example to illustrate the behavior. The example chosen corresponds to a temporally-growing mixing layer. The computation uses nonreflecting boundary conditions based on characteristic variables. These allow the acoustic waves to leave the computational domain. The example discussed here has $M_{c}=0.6$, a value for which we find significant departures from the low Mach number aero-acoustic theory. The computational domain contains two wavelengths of the most unstable disturbance. The layer first rolls up to form two vortices which later pair to form a larger vortex. After the pairing the vortex continues to undergo shape oscillations for several cycles. This behavior is illustrated in figure 12 where the time history of layer thickness is plotted. The corresponding time history of pressure, velocity component normal to the layer, and the acoustic flux of energy leaving the domain near the bottom are

also shown in this figure. Pressure is normalized as $\frac{p / p_{\infty}-1}{M_{c}^{3}}$, velocity as $\frac{v / a_{1}}{M_{c}^{3}}$, and acoustic flux in terms of these normalized values. The roll up, pairing and nutation processes can be identified in these figures. The roll up and pairing cause the layer thickness to increase and generate a compression wave. Subsequent to pairing, the nutation of the vortices causes a periodic energy exchange between the vortices and the mean flow. This process generates a series of compression and expansion waves which carry acoustic energy away from the layer. The numerical simulations show that the nutation frequency is close to $\frac{\omega}{2}$, where $\omega$ is the peak vorticity.

In figure-13 the acoustic radiation for three different cases are compared. All case were for $M_{c}=0.4$, but differed in the initial disturbance. Case-1 contained only the fundamental disturbance (case-1), case-2 only the subharmonic disturbance, and case-3 contained both the fundamental and the subharmonic. It is evident (as anticipated by Laufer (1974)) that in case-3 the vortex merger produces the strongest acoustic radiation ( 8 times that in case-1 and twice that of case-2). In the time history of the acoustic signal the signals arising from the rollup and pairing can be separately identified. The acoustic signal from rollup 
contains a compression wave followed by a more spread out expansion wave while the acoustic signal from pairing generates more symmetric compression and expansion waves.

\section{Simulations with random initial conditions}

Temporally evolving mixing layer flows were simulated starting from very low level (peak amplitude $10^{-4}$ ) white noise (equal amplitude to all fourier modes with uncorrelated phases). Both two-dimensional and three-dimensional simulations were conducted. The computational box was chosen to be 12 times the most unstable wavelength (based on the initial profile). The initial evolution of the disturbances corresponded to the predictions of the linear stability analysis. In figure-14 the exponential growth rates obtained from two such simulations are shown. The two cases correspond to $M_{c}=0.4$ and $M_{c}=0.8$. The wavenumber and the growth rate are scaled with the local values of the vorticity thickness. The time scale is normalized by $\frac{\delta_{w}}{U_{1}-U_{c}}$ in this figure. During this phase of exponential amplification the layer grew in a laminar fashion. As the disturbances became nonlinear the harmonics of the linearly unstable disturbances began to amplify. With significant vorticity clumping the growth rates became larger than the 'laminar instability' estimates. The nonlinear process generated a broadband spectrum. In this regime a linear spreading of the mixing layer was observed. In figure-15 data from three simulations are presented. It may be seen that for $M_{c}=0.4$ and $M_{c}=0.6$ a constant spreading rate was observed, with slower growth for $M_{c}=0.6$. It was verified that for $M_{c}$ lower than 0.4 the growth rate was not further increased. The temporal growth rate obtained for $M_{c}=0.4$ is close to 0.1 a value consistent with previous incompressible simulations Lesieur (1987). For $M_{c}=0.8$ the growth was slow and dominated by a laminar process. Later in time (outside the time range shown) vortices formed in the layer but the computational domain contained too few of them to constitute an adequate statistical sample. The convective Mach number concept was also tested with random initial conditions. In figure-16 the time evolution of two run with the same $M_{c}$ of 0.4 but different density ratios are compared. The temporal growth rates were quite close (though not identical).

In figure-17 snapshots of the vorticity field are displayed for the case with $M_{c}=0.4$. The frames are equally separated in time. It may be seen that the linear instability process selects a length scale for the vortices. This length scale does not correspond to the most unstable wavelength of the initial profile. This is because the initial disturbance level was very low, allowing the layer to viscously thicken $\left(R e_{\delta_{\omega_{0}}}=400\right)$. The length scale chosen by the flow corresponds to the most unstable wavelength at a later time (end of the laminar spreading regime in fig. 15). After the vortices form they undergo the merging instability. Since the vortices had a phase jitter the merging is also randomized. Figure-18 presents similar snapshots for the mixing layer at $M_{c}=0.6$. It maybe noted that the length scale selected by the flow is larger. This is consistent with the 
linear instability behavior. The phase jitter in the vortices is again visible. Such phase jitter allows the turbulence statistics to evolve in a self-similar manner. Figure-19 presents some of these statistics at four instants in the evolution (from the linearly spreading regime). The y-coordinate in the plots is scaled with the instantaneous vorticity thickness and the fluctuations are scaled by half the velocity difference across the layer. The mean velocity profiles are closely selfsimilar. The Reynolds stress is also close to being self-similar. The r.m.s. $u$ and r.m.s. $v$ (titled as u prime and $v$ prime) have larger departure from self-similarity. Later in the evolution as the second pairing takes place the computational box contains too few vortices ('independent' samples) and the layer departs from self-similarity. As noted earlier the nonlinear process generate higher harmonics and create a broadband spectra. In figure-20 spectra of the fluctuating fields are plotted at various times. The spectra were obtained by fourier decomposing the fields in the periodic direction. The wavenumber was normalized with the initial vorticity thickness. The plots show the spectra obtained by integrating the energy associated with each fourier mode across the layer. The simulation start from a low level white noise. Linear instability selectively amplifies the unstable modes. Later in time the spectra fill up in the harmonics and finally become broadband. It is possible to see a shift in the peak of the spectra to low wavenumbers (since the layer grows in time). Even at late times the energy level of the broadband fluctuations is quite low. Previous studies of incompressible mixing layer (Lesieur, 1987) have suggested a -4 slope in the broad band portion. Such a spectral slope may be seen in the present simulations. Interestingly the low wavenumber portion of the pressure spectrum is very much like the velocity spectrum, but at higher wavenumber the pressure spectrum drops more rapidly.

This work was presented at the AIAA meeting in Reno, 1989. It is available as AIAA paper AIAA-89-0374 from AIAA.

\section{REFERENCES}

BOGdANOFF, D. W. 1983 AIA A J., 21, 926-927.

BORIS, J. P 1988 in Proceedings of the conference on 'Physics of Compressible Turbulent Mixing', Princeton. (see also Oran, E.)

Brown, G. L. AND RoshKo, A. 1974 J. Fluid Mech., 64, 775-816.

CENTER FOR TURBULENCE RESEARCH 1987 Studying Turbulence using Numerical Simulation Databases, Proceedings of the 1987 Summer Program, Report CTR-S87, Stanford University.

Center for TURbUlence RESEARCH 1988 Studying Turbulence using Numerical Simulation Databases - II, Proceedings of the 1988 Summer Program, Report CTR-S88, Stanford University.

Corcos, G. M. And Sherman, F. S. 1984 J. Fluid Mech., 139, 29-65. 
CoRcos, G. M. AND LIN, S. J. 1984 J. Fluid Mech., 139, 67-95.

DIMOTAKIS, P. E. 1984 AIAA paper, AIAA-84-0368.

Feiereisen, W. J., Reynolds, W. C. AND Ferziger, J. H. 1981

Dept. of Mech. Engrg., Stanford University, Report No. TF-13.

KIM, J., MOIN, P. AND MOSER, R. D. 1987 J. Fluid Mech., 177, 133-166.

LAUfER, J. 1974 Omaggio a Carlo Ferrari, 451-464.

LEe, M. J. AND ReYnolds, W. C. 1985 Dept. of Mech. Engrg., Stanford University, Report No. TF-24.

LELE, S. K. 1988a submitted to J. Comput. Phys.

LELE, S. K. 1988b in Proceedings of the conference on 'Physics of Compressible Turbulent Mixing', Princeton.

LELE, S. K. AND HO, C. M. 1989 in preparation.

Lesieur, M. 1987 Turbulence in fluids, M. Nijhoff Publishers, Chapter 8, p253.

LOWERy, P. S. AND REYNoldS, W. C. 1986 Dept. of Mech. Engrg., Stanford University, Report No. TF-26.

Mansour N. N., KIM, J. AND MoIN, P. 1988 J. Fluid Mech., 194, 15-44. METCALFE ET. AL. $1987 \mathrm{~J}$. Fluid Mech., 184, 207-243.

MoIn, P. AND SPalarT, P. R. 1987 NASA TM-100022.

MOSER, R. D. AND MOIN, P. 1987 J. Fluid Mech., 175, 479-510.

PAPAMOSCHOU, D. AND ROShKo, A. 1986 AIAA paper, AIAA-86-0162.

PAPAMOSCHOU, D. 1988 in Proceedings of the conference on 'Physics of Compressible Turbulent Mixing', Princeton.

PASSOT, T. AND POUQUET, A. 1987 J. Fluid Mech., 181, 441-466.

RAGAB, S. A. AND WU, J. L. 1988 AIAA paper, AIAA-88-0038.

RILEY, J. J. AND METCALFE, R. W. 1980 AIAA paper,

ROGALLO, R. 1981 NASA TM-81315.

Rogers, M. M., MoIn, P. ANd ReYNolds, W. C. 1986 Dept. of Mech. Engrg., Stanford University, Report No. TF-25.

SAMIMY, M. AND ELLIOTT, G. S. 1988 AIAA paper 88-3054A.

SANDhAM, N. D. AND REYNoldS, W. C. 1989 AIAA paper, AIAA-890371.

SANDhAM, N. D. AND ReYNolds, W. C. 1987 in Turbulent Shear Flows $V I$, Toulouse, France.

SMARR, L. L., NORMAN, M. L. AND WINKLER, K-H. A. 1984 Physica $D, 12,83-106$. 
Soetrisno, M., Eberhardt, S., Riley, J. J., and McMurty, P. 1988 AIAA paper, AIAA-88-3676.

SPALART, P. R. 1988 J. Fluid Mech., 187, 61-98.

Thompson, K. W. 1987 J. Comput. Phys., 68, 1-24.

WINKLER ET. AL. 1987 Phys. Today, October, 28-37.

WOODWARD, P. 1984 L. L. L. preprint UCRL-90662.

Wray, A. A. 1987 submitted to J. Comput. Phys.

Zhuang, M., Kubota, T. AND Dimotakis P. 1988 in Proceedings of the $1^{\text {st }}$ National Fluid Dynamics Conference, Cincinnati. 88-3583-CP. 


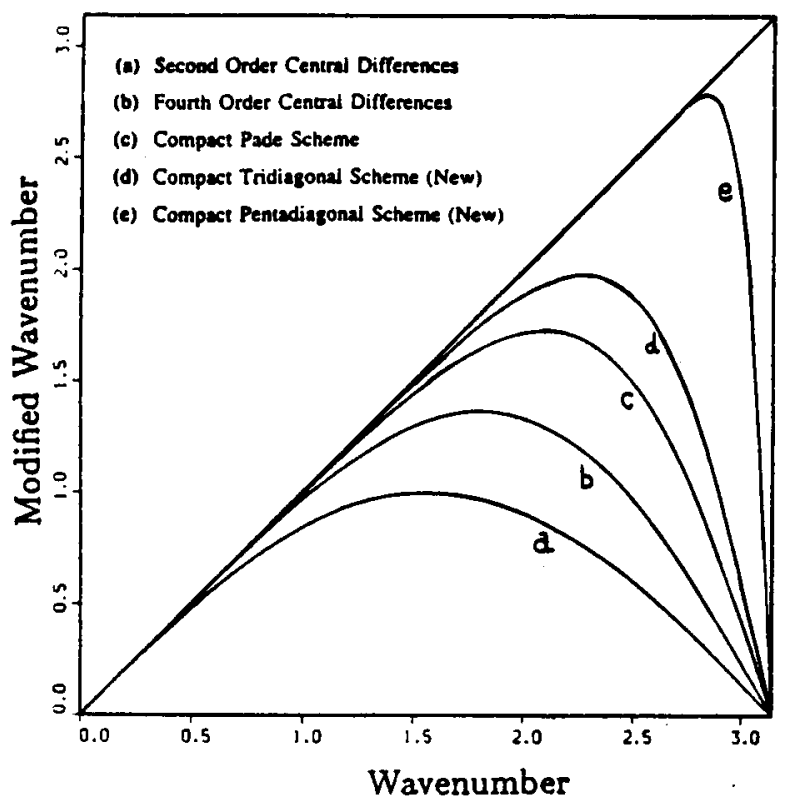

Figure 1 Modified warenumber analysis for the approximations to the first derivative.

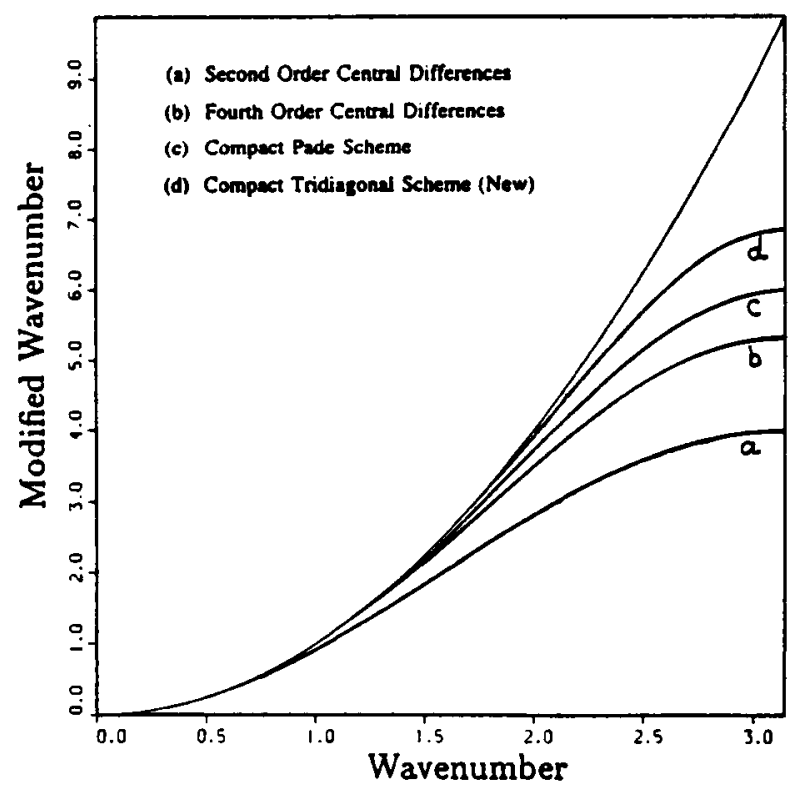

Figure 2 Modified wavenumber analysis for the approximations to the second derivative.
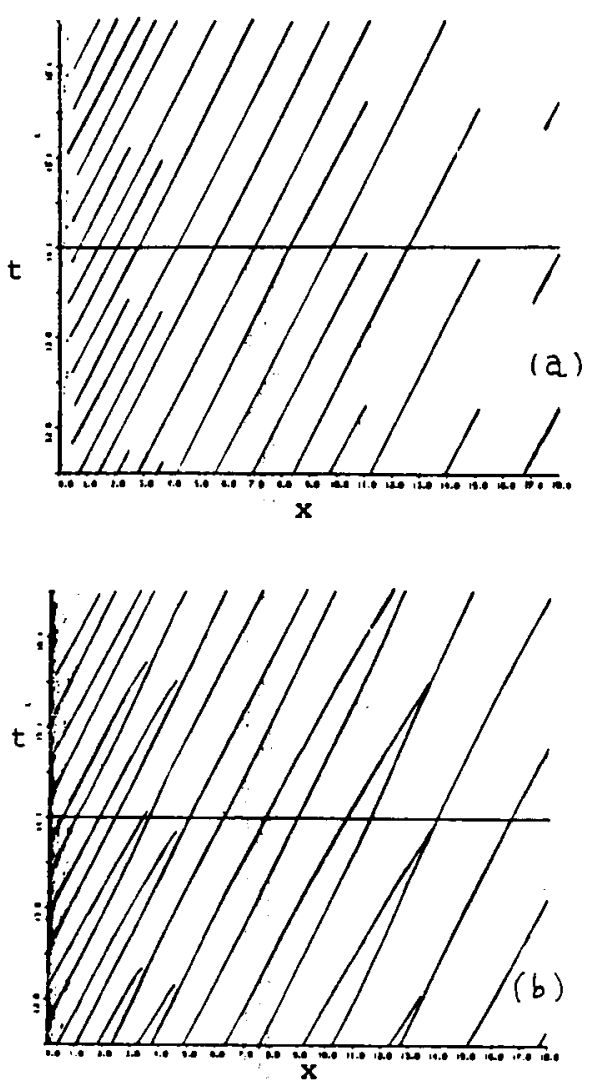

Pigure 3 Trajectories of pressure extrema in a spatially erolving mixing layer. Pressure maxima shown in a) correspond to the stagnation points in the convected frame and pressure minime shown in b) correspond to the vortex centers. 


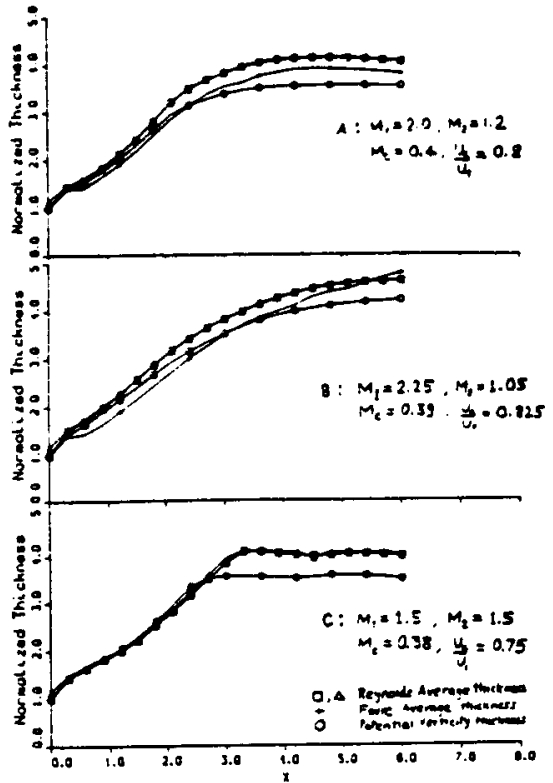

Figure 4 Streamwise erolution of mixing layer thickness measures. All cases ba:"e $U_{2}$ of 0.6 , $M_{c}$ abont 0.4 and the same inflow forcing. The difference in the growth rates is due to the different density ration. A) $T_{1}=T_{2}$, B) $T_{0_{1}}=T_{0,}$, C) $M_{1}=M_{2}$.
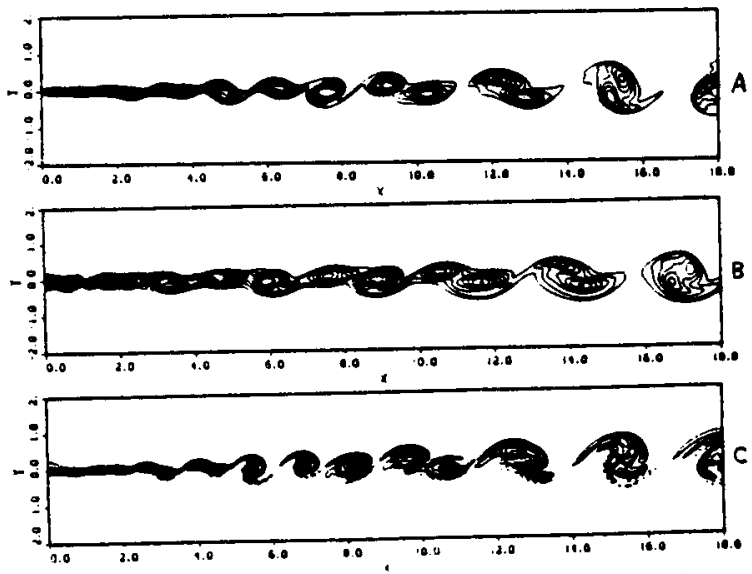

Figure 5 Vorticity contours in spatially evolving mixing layers. All cases have $U_{2}$ of $0.6, M_{c}$ about 0.4 and the same intor forcing. $A$ ) $T_{1}=T_{2}$, B) $T_{0}=T_{0}$, C) $M_{1}=M_{2}$.

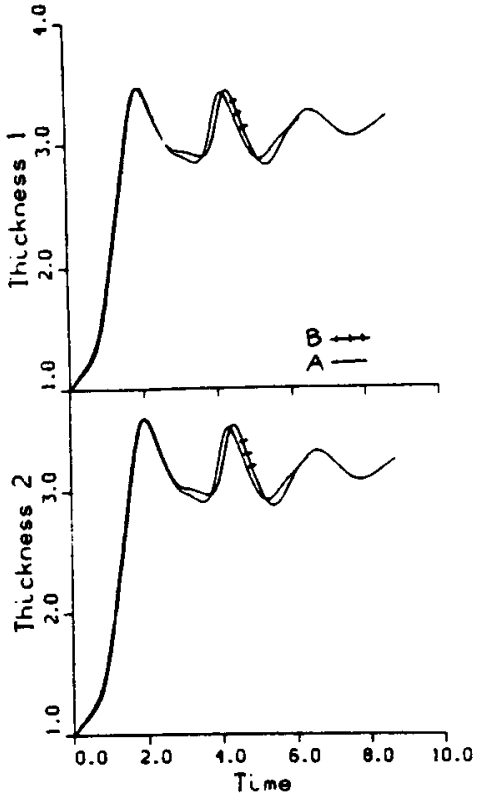

Figure 6 Temporal evolution of mixing layer thickness measures. Both cases have the same $M_{c}=$ 0.38, A) $T_{1}=T_{2}$, B) $T_{0,}=T_{0,}$.

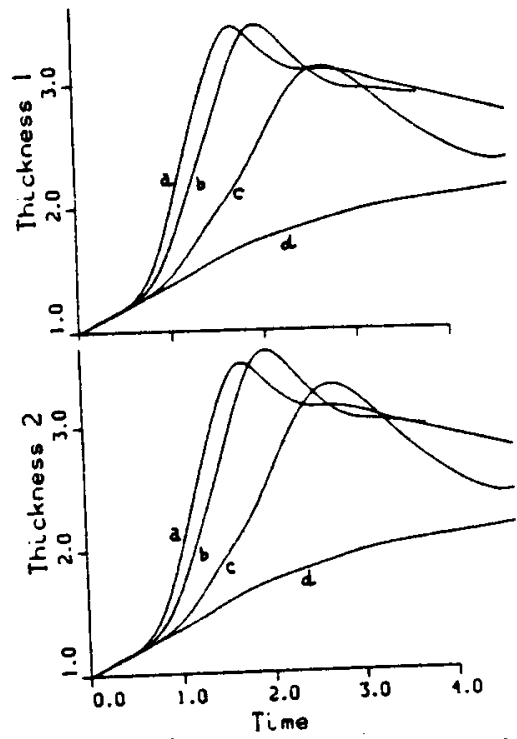

Figure 7 Temporal evolution of mixing layer thickness neasures for different $M_{c}$. All cases are from claes $A$ : a) $M_{e}=0.2$, b) $M_{c}=0.38$, c) $M_{c}=0.6$, d) $M_{c}=0.8$. 


\section{ORIGINAL PAGE IS \\ OF POCR QLALITY}

Direct numerical simulation of compressible free shear flows
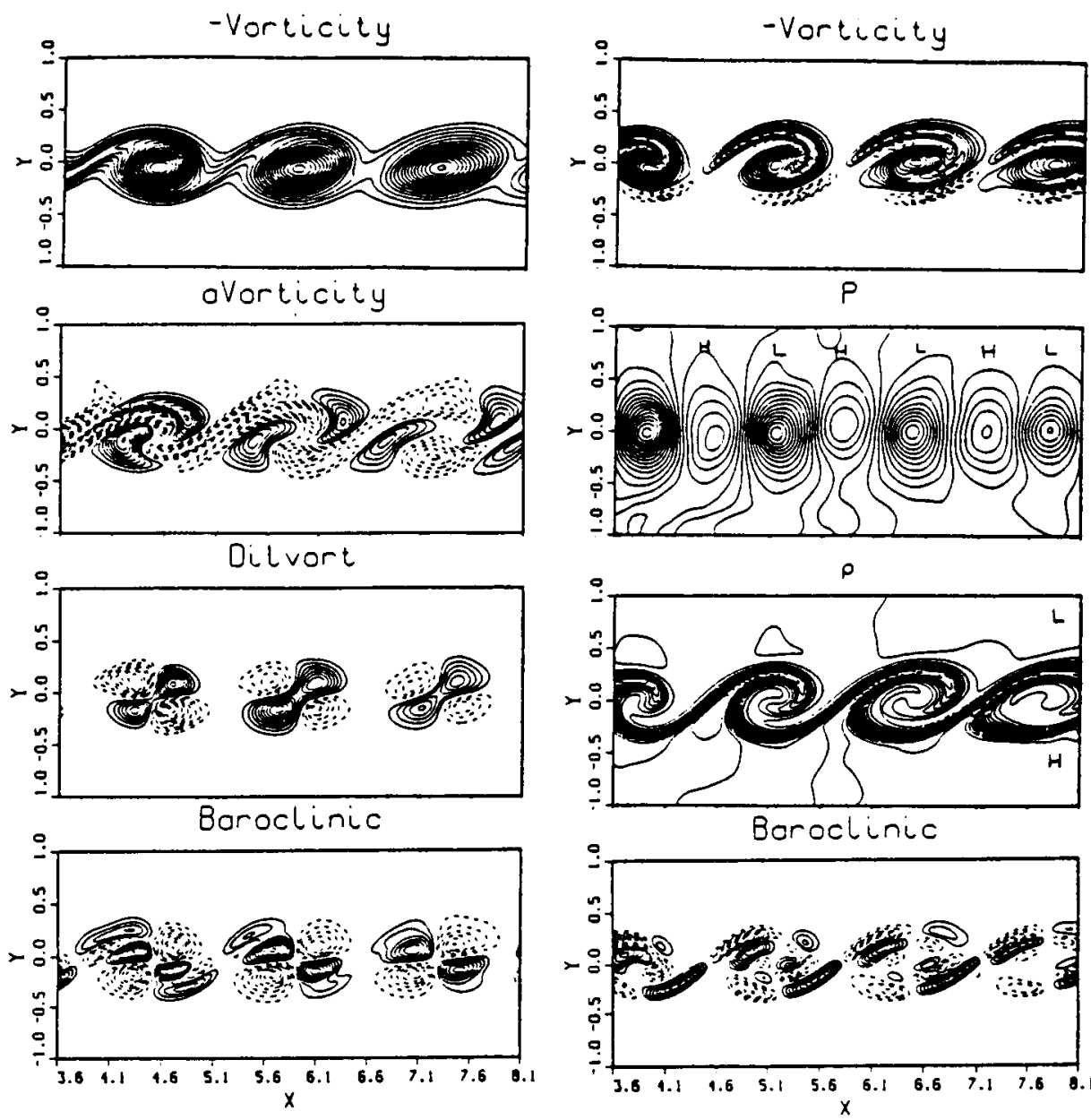

Figure 8 Vorticity evolution for class A mixing layer. $M_{1}=2.0, M_{2}=1.2, M_{c}=$ 0.4 . The panels are : vorticity field. advection term in moving frame (0.91 to 0.76$)$, vorticity compression term $(-0.22$ to 0.25$)$, and baroclinic term $(-0.03$ to 0.04$)$, respectively.

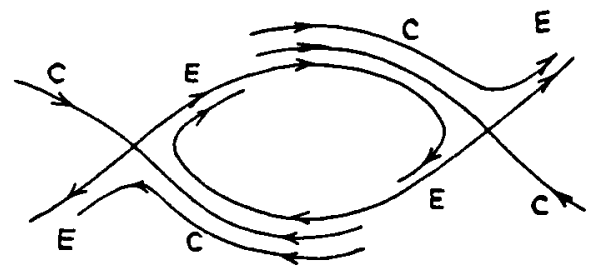

Figure 9 Vorticity evolution for class $\mathrm{C}$ mixing layer. $M_{1}=1.5, M_{2}=1.5$, $M_{c}=0.38$. The baroclinic terms are comparable to the vorticity advection and produce the layered vorticity profiles. The panels are : vorticity field, pressure field, density field, and the baroclinic term, respectively.

$$
\begin{gathered}
\text { E : Expansion } \\
\nabla \cdot u>0 \\
\text { C: Compresion } \\
\nabla \cdot u<0
\end{gathered}
$$

Figure $10 \mathrm{~A}$ schematic of the flow field in a frame of reference moving with the vortices. 

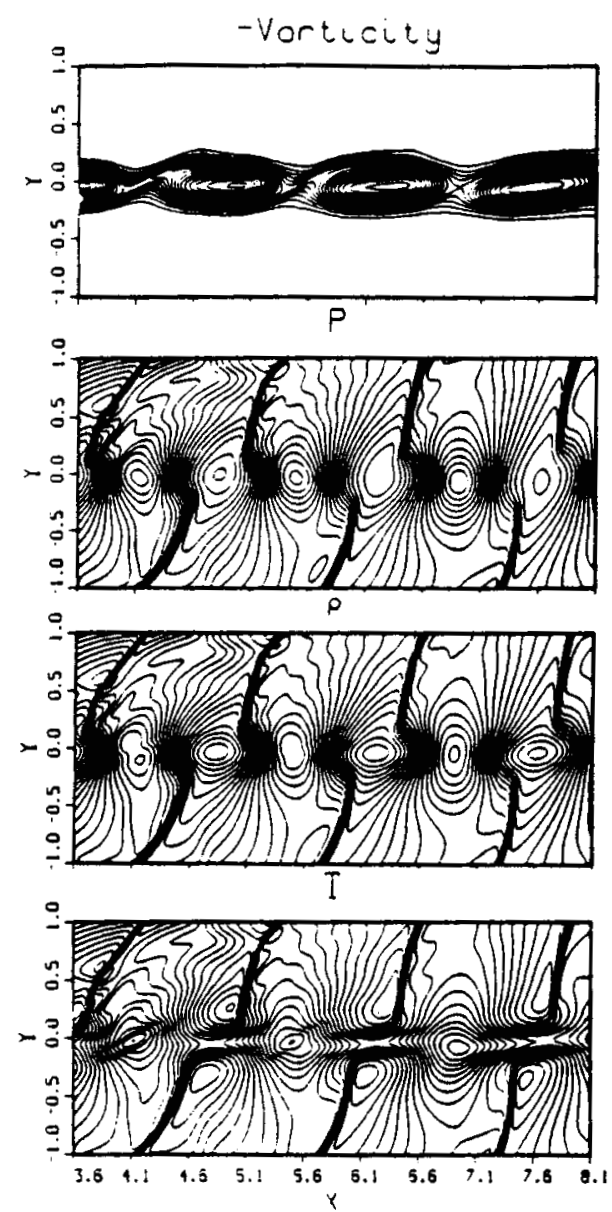

Pigure 11 Vortex evolution with eddr shocklets. The example shown is for class A mixing layer. $M_{1}=4.0, M_{3}=2.4, M_{e}=0.8$. The pan:ls present the zortieity field, pressure field, density field, and temperature field, respectively.
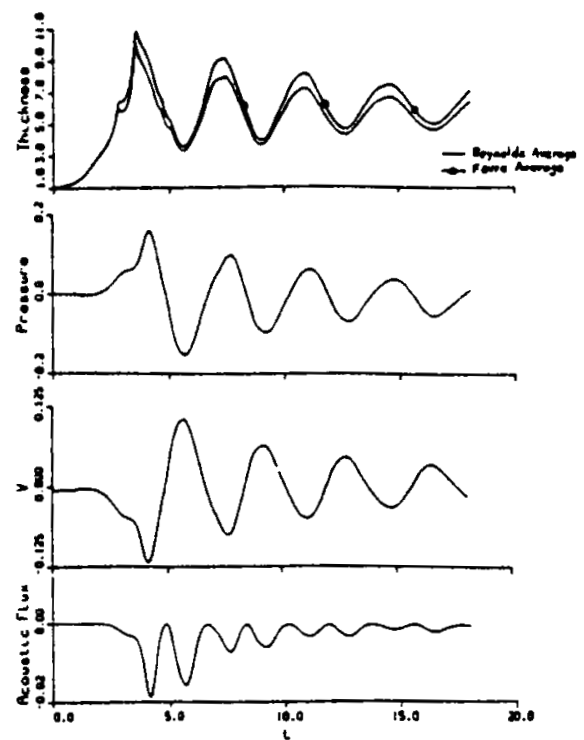

Figure 12 Acoustic rediation from rortex pairing and shape oucillation. $M_{c}=0.6$, Time history of mixing layer thickness measures, normalised preasure, normalised $\mathbf{v}$ velocity, and normalised acoutic flux at the bottom boundary.
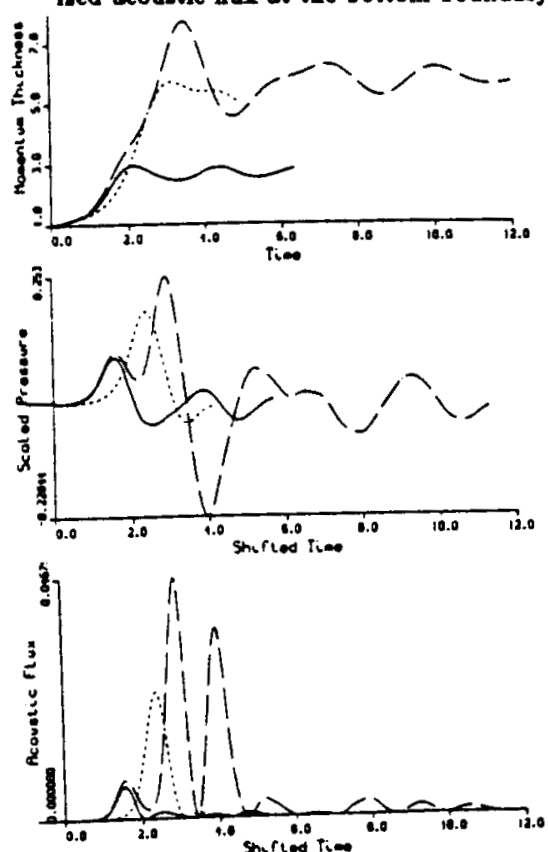

Figure 13 Acoustic radiation from rortex evolution. All eases have $M_{c}=0.4$. Case-1 (solid) has only the fundamental disturbance, 2) only the subharmonic disturbance (dashed) and 3) both togather (chain). The normalised preseure signal in the far-field and normalised acoustic finx at the top boundary are shorn. 

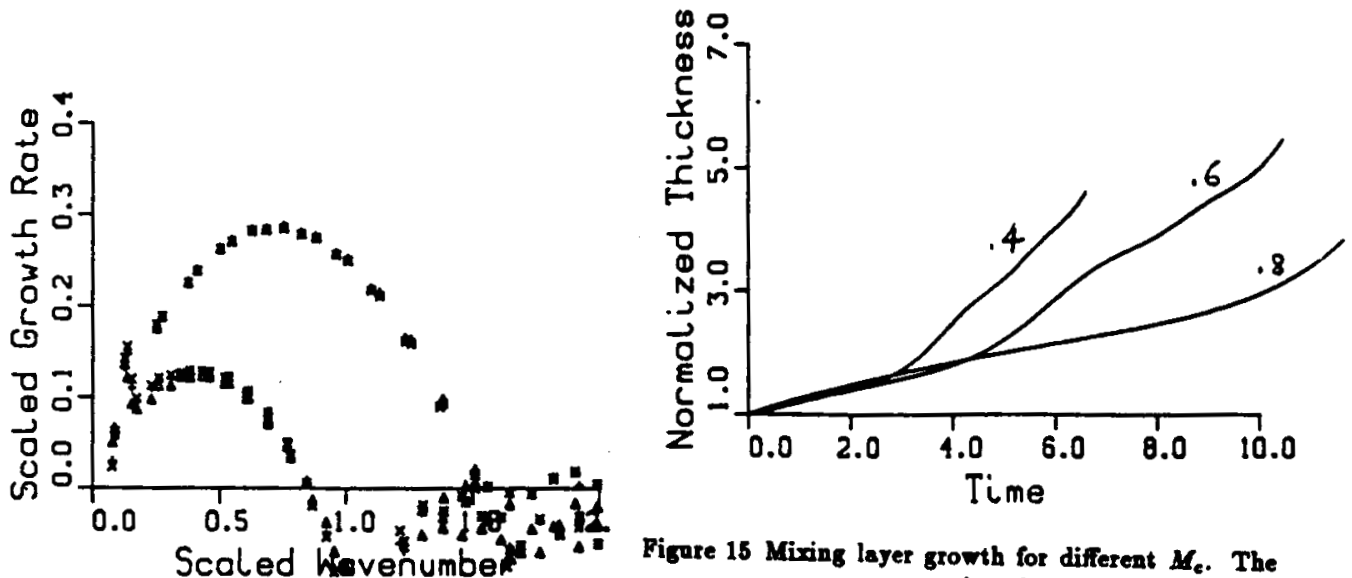

Figure 15 Mixing leyer growth for difierent $M_{\varepsilon}$. The eurves correspond to $M_{c}=0.4,0.6$ and 0.8 .

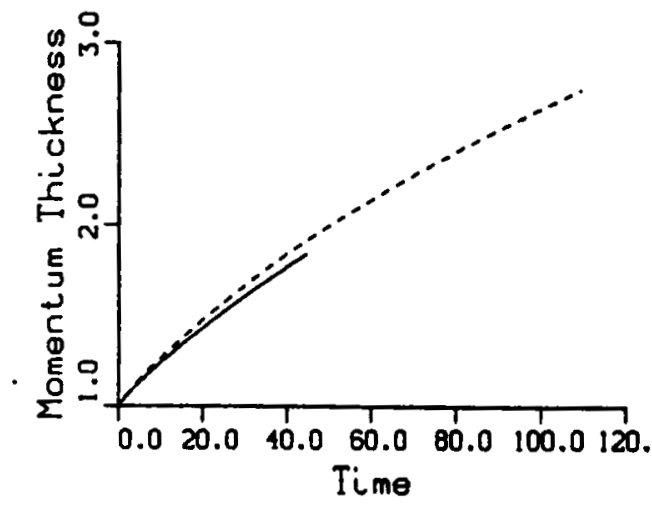

Figure 14 Growth rates for different fourier modes for $M_{c}=0.4$ (solid) and $M_{\epsilon}=0.8$ (dashed). During this phase laminas spreading is observed.

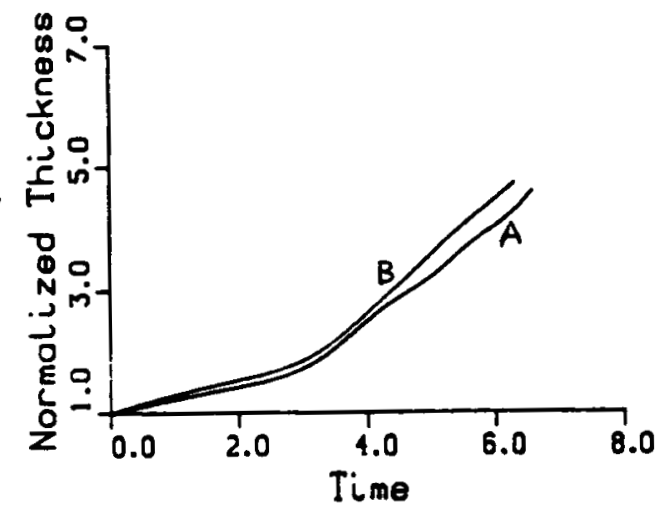

Figare 16 Miving layer growth at $M_{c}=0.4$. A) $T_{1}=$ $T_{3}$, B) $T_{0_{1}}=T_{0_{2}}$. 


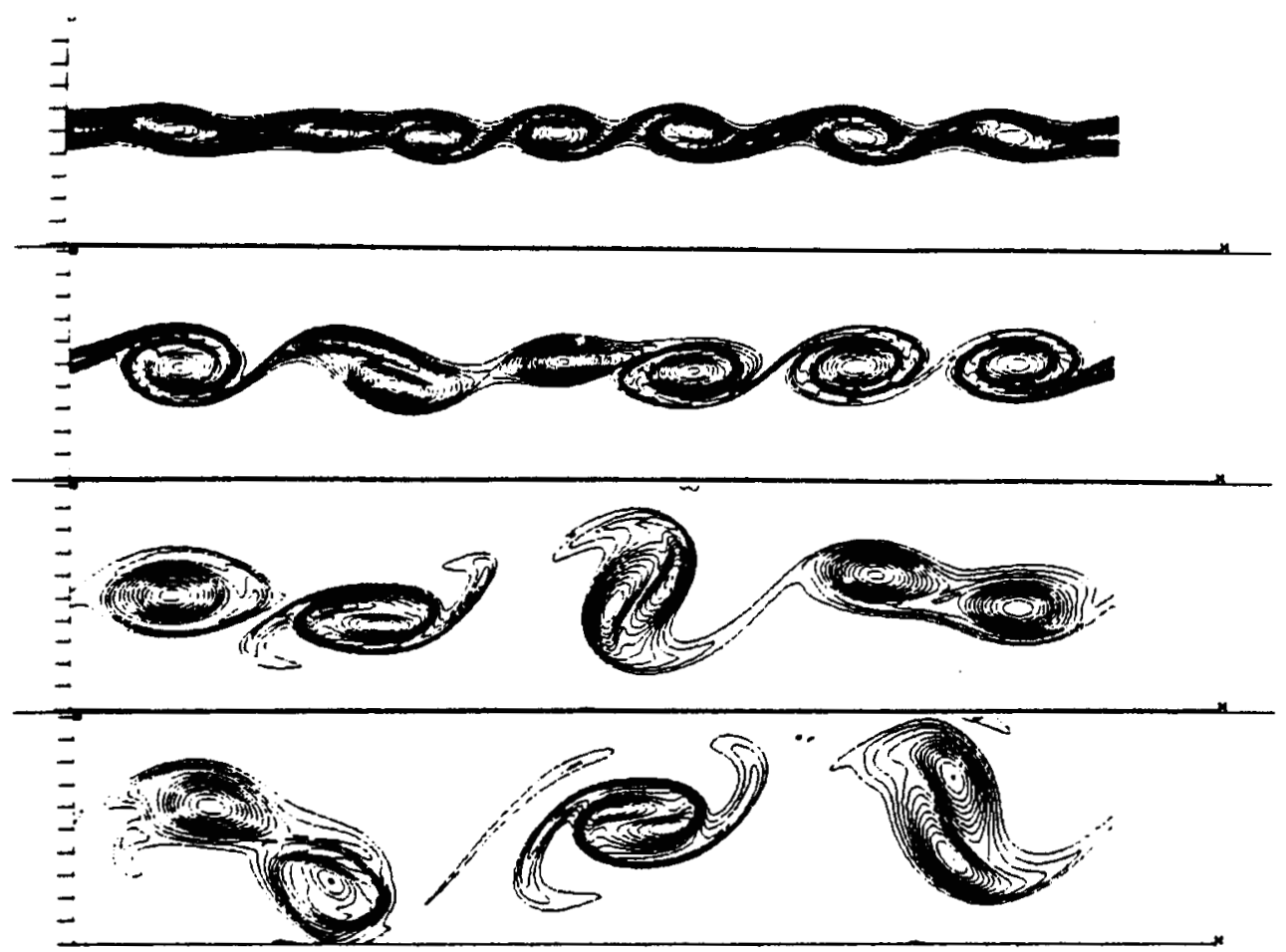

Figure 17 Snap shots of mixing layer evolution at $M_{\epsilon}=$ 0.4. Contours of vorticity are shown.
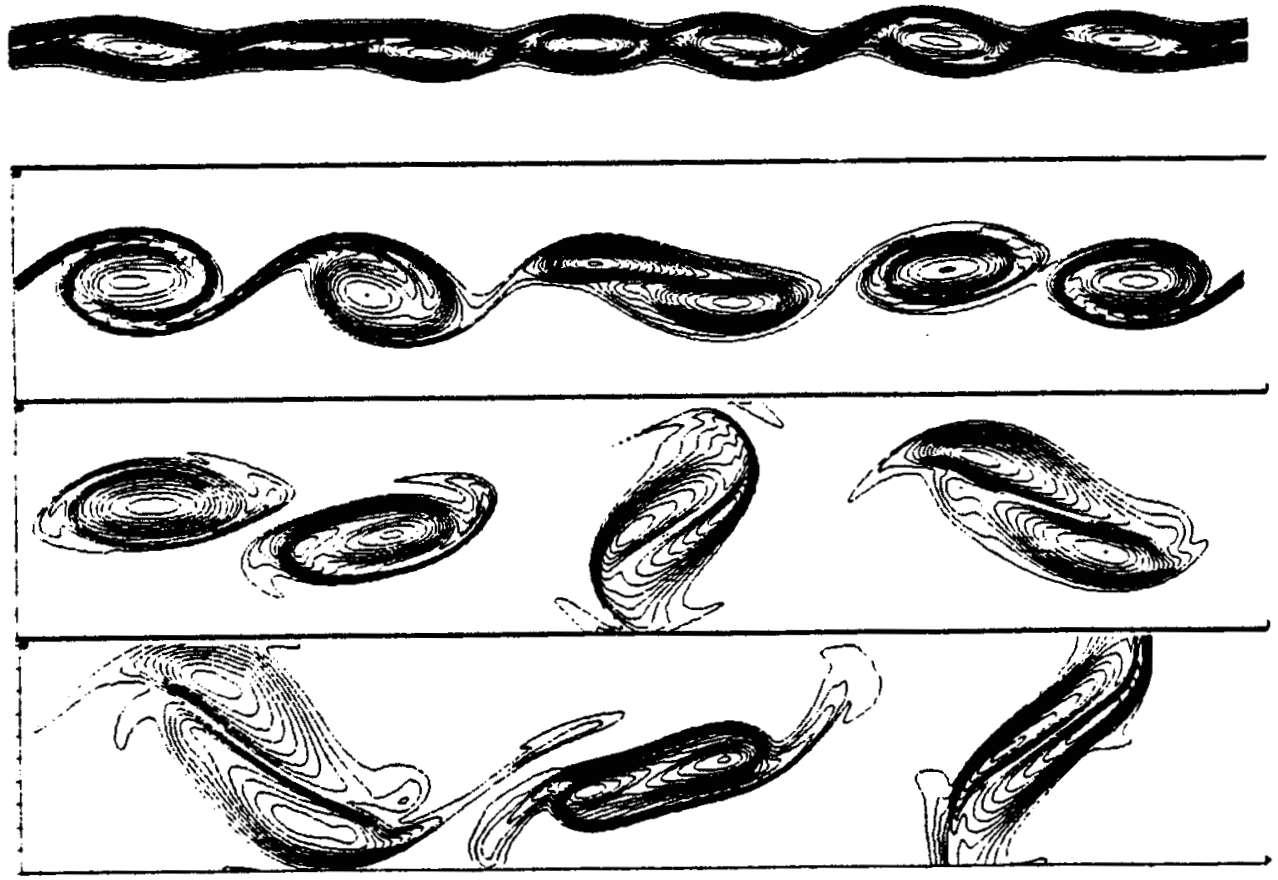

Figure 18 Snep shots of mixing layer evolution at $M_{c}=$ 0.6. Contours of rorticity are shown. 

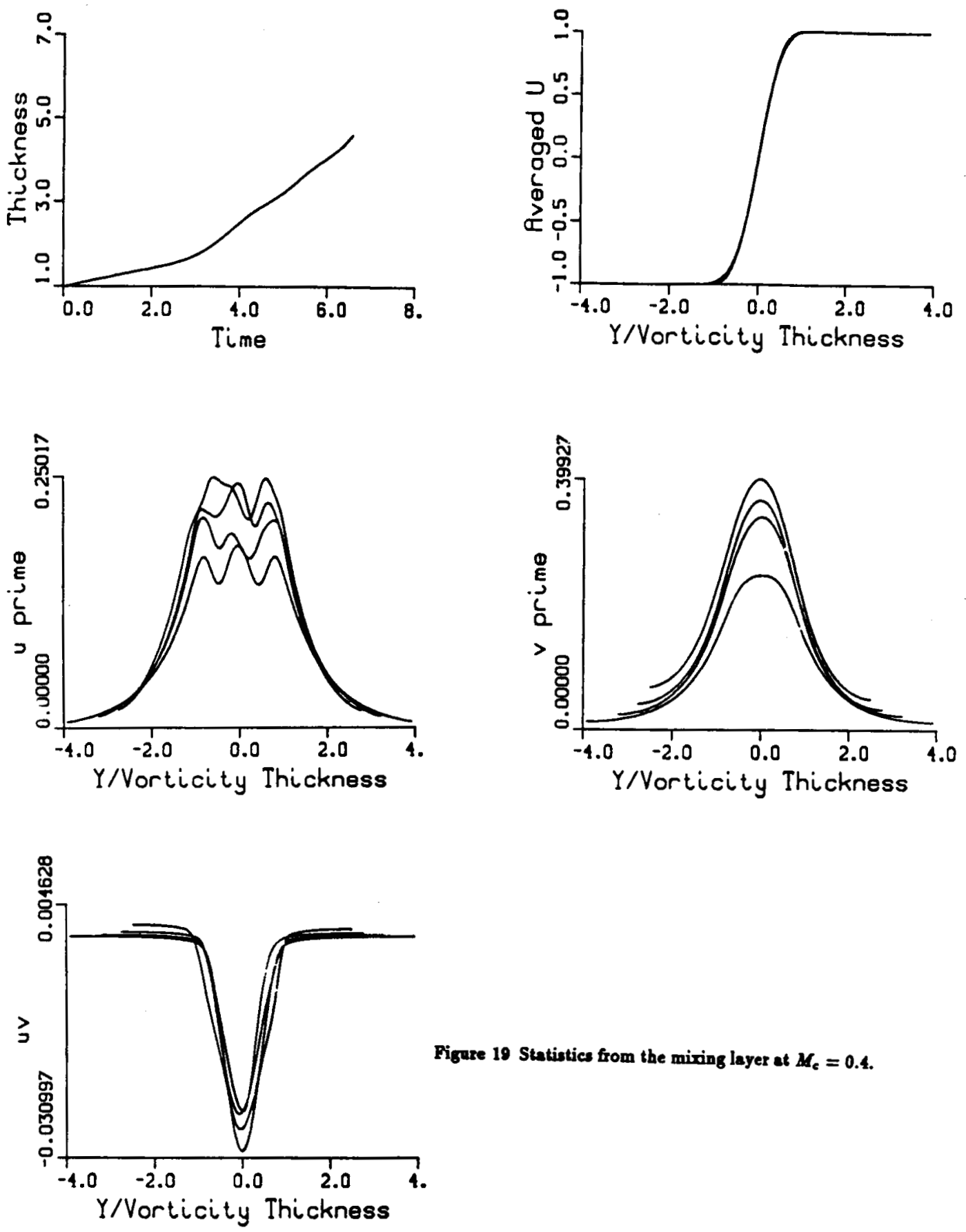

Figare 19 Statistics from the mixing layer at $M_{c}=0.4$. 
98

S. K. Lele
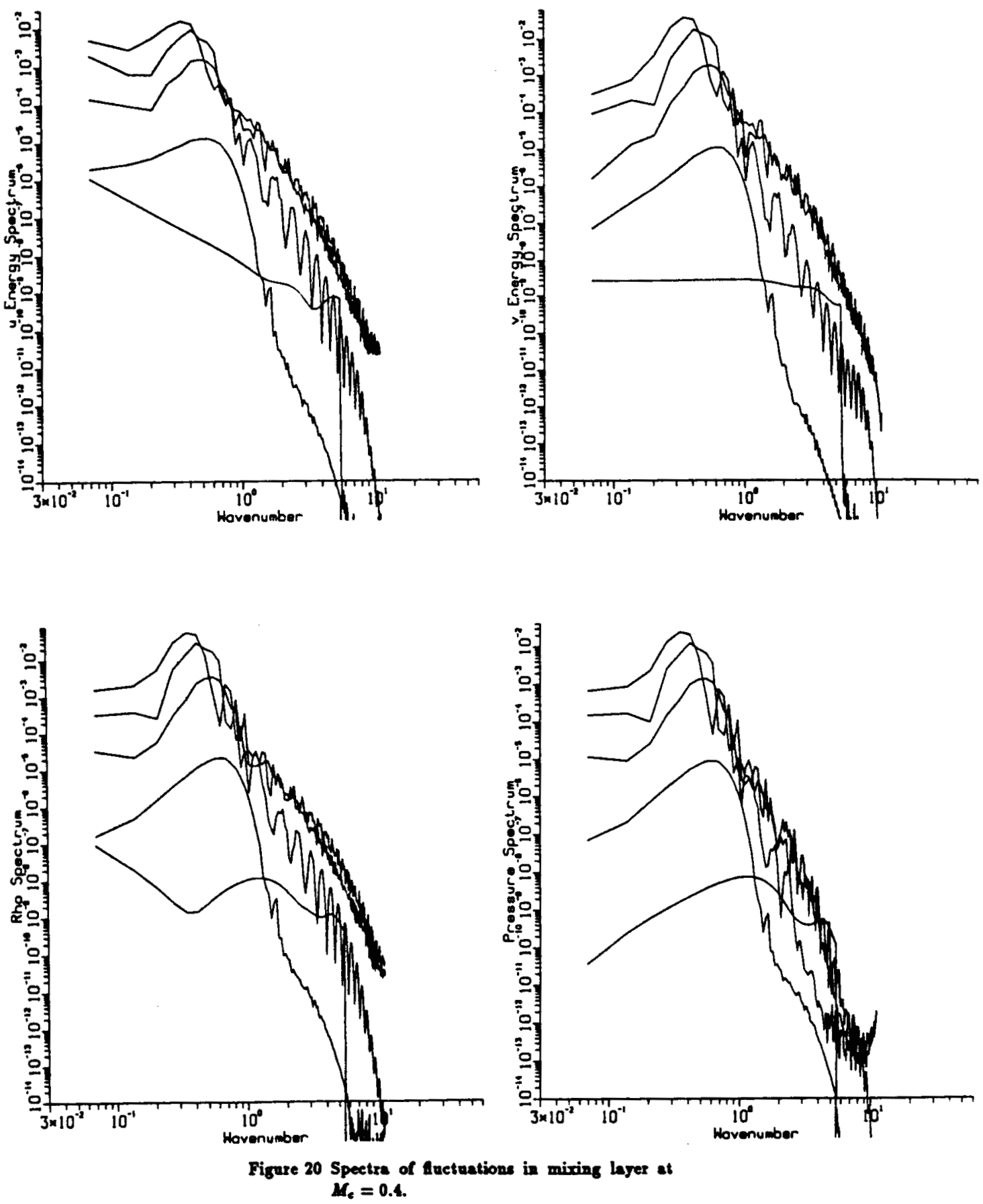